\title{
Management of idiopathic pulmonary fibrosis: selected case reports
}

\author{
Michael Kreuter ${ }^{1}$, Peter Kardos ${ }^{2}$ and Victor Hoffstein ${ }^{3}$ \\ Affiliations: 'Dept of Pneumology and Respiratory Critical Care Medicine, Thoraxklinik, University of \\ Heidelberg, Heidelberg, Germany. ${ }^{2}$ Group Practice and Respiratory, Allergology and Sleep Unit, Maingau
} Hospital, Frankfurt, Germany. ${ }^{3}$ Division of Respirology, St. Michael's Hospital, Toronto, Canada.

Correspondence: Michael Kreuter, Dept of Pneumology and Respiratory Critical Care Medicine, Thoraxklinik, University of Heidelberg, Amalienstrasse 5, D-69126 Heidelberg, Germany.

E-mail: kreuter@uni-heidelberg.de

ABSTRACT In 2011, revised international guidelines were issued jointly by the American Thoracic Society, the European Respiratory Society, the Japanese Respiratory Society and the Latin American Thoracic Association, which provide a valuable framework for the diagnosis and management of idiopathic pulmonary fibrosis (IPF). However, due to the complexity of IPF, these guidelines may not comprehensively account for the management of individual IPF patients in clinical practice.

We describe three patient cases that were presented and discussed during the 2013 AIR: Advancing IPF Research meeting in Nice, France. These cases highlight the heterogeneity in the presentation, history and clinical course of IPF, together with expert insights regarding the diagnosis and management of IPF in the real-life setting.

○

@ERSpublications

IPF is the most common of the idiopathic interstitial pneumonias and is associated with poor survival http://ow.ly/v738f

\section{Introduction}

Idiopathic pulmonary fibrosis (IPF) is a chronic lung disease in which progressive fibrosis and scarring of the lung parenchyma leads to symptoms such as dyspnoea on exertion, dry cough and, ultimately, respiratory failure [1]. IPF is a complex disease with several aspects that are not fully understood, including its pathogenesis and variable clinical course [1-4].

The 2011 American Thoracic Society (ATS)/European Respiratory Society (ERS)/Japanese Respiratory Society (JRS)/Latin American Thoracic Association (ALAT) guidelines are a clear step forward in the diagnosis and treatment of IPF [5]. However, due to the complex course of this disease they do not comprehensively represent the management of all individual IPF patients. Moreover, in the 2011 ATS/ERS/ JRS/ALAT guidelines, no pharmacological therapy received a positive recommendation. As new results from clinical trials have been published after these guidelines and with anticipated new insights into the treatment of IPF, an update of these recommendations is highly warranted in the near future. Currently, the only approved drug for the treatment of mild-to-moderate IPF within the European Union is pirfenidone, which is also approved for IPF in Canada, Japan, India and China [6, 7].

The multifaceted clinical course of IPF in individuals and the lack of updated guidelines on pharmacological management can present a number of challenges to the clinician in the real-life setting. Herein, we report

Received: March 102014 | Accepted after revision: March 142014

Conflict of interest: Disclosures can be found alongside the online version of this article at err.ersjournals.com

Provenance: Publication of this peer-reviewed article was sponsored by InterMune International AG, Muttenz, Switzerland (article sponsor, European Respiratory Review issue 132).

Copyright OERS 2014. ERR articles are open access and distributed under the terms of the Creative Commons Attribution Non-Commercial Licence 4.0. 
three case studies presented during the 2013 Advancing IPF Research (AIR) meeting in Nice, France, which provide insights into the complex management of IPF from a real-life perspective.

\section{Case 1}

\section{Complex diagnosis of IPF: a case-based discussion}

The recommendations of the 2011 ATS/ERS/JRS/ALAT evidence-based guidelines apply to patients typically represented in clinical trial populations [5]. The presentation and course of IPF is, however, highly individual and the utility of these guidelines in the real-life setting is sometimes limited. Thus, to ensure effective treatment, patients with suspected interstitial lung diseases (ILDs) need multidisciplinary team (MDT) evaluation.

A 55-year-old female who was a current smoker presented in general practice with a 9-month history of respiratory symptoms, including dyspnoea on exertion, thoracic pain and dry cough, which were preceded by a pulmonary infection. Pulmonary function tests (PFTs) showed only mild impairment of vital capacity (VC) but a severely reduced diffusing capacity of the lung for carbon monoxide (DLCO). In accordance with the diagnostic algorithm for patients with suspected ILD [8], a chest multi-slice high-resolution computed tomography (HRCT) scan was performed (fig. 1 and table 1). A number of findings were noted on the scan: 1) apical, paraseptal emphysema; 2) reticular abnormalities and honeycombing with traction bronchiectasis (predominantly in the subpleural and basal regions); and 3) extensive ground-glass opacities. According to the current guidelines, due to the ground-glass opacities a diagnosis of IPF could not be made [5].

The patient was unable to undergo surgical lung biopsy (SLB) due to the severe functional impairments and, following a detailed MDT discussion, the patient was advised to stop smoking. A short-term follow-up of the patient was initiated. On examination 3 months later and after smoking cessation, the ground-glass opacities were significantly reduced on HRCT, while DLCO increased to $51 \%$. To better define the diagnosis a further MDT meeting was held and it was decided to perform SLB. Histopathological evaluation of the biopsy demonstrated a typical usual interstitial pneumonia (UIP) pattern and emphysematous changes. In addition, there was diffuse pulmonary involvement of numerous macrophage accumulations within most of the distal airspaces, consistent with a desquamative interstitial pneumonia (DIP) pattern. Following a final interdisciplinary discussion, the patient was diagnosed with combined pulmonary fibrosis and emphysema in combination with another smoking-related disease, namely DIP (fig. 2) [9].

The patient also had a number of comorbidities, including gastro-oesophageal reflux disease (GORD), a prior transient ischaemic attack and depression. A holistic management approach was discussed with the patient and then implemented. The plan involved initiation of pharmacological treatment with pirfenidone for mild-to-moderate IPF, a proton-pump inhibitor (PPI) for GORD and an antidepressant. The patient was also vaccinated for influenza and pneumococci and referred to an outpatient rehabilitation centre. In addition, information regarding patient support groups was provided as were supportive measures to help the patient maintain her smoking cessation.

Pirfenidone treatment resulted in a period of stabilisation; however, this was followed by a gradual decline and eventually a sharp decrease in the patient's forced vital capacity (FVC). Subsequent investigations ruled out an acute exacerbation. The patient was further evaluated and it was then discovered that the patient had a neurological problem which led to back pain. The patient underwent surgery and following this there was a marked improvement in her FVC (fig. 3).
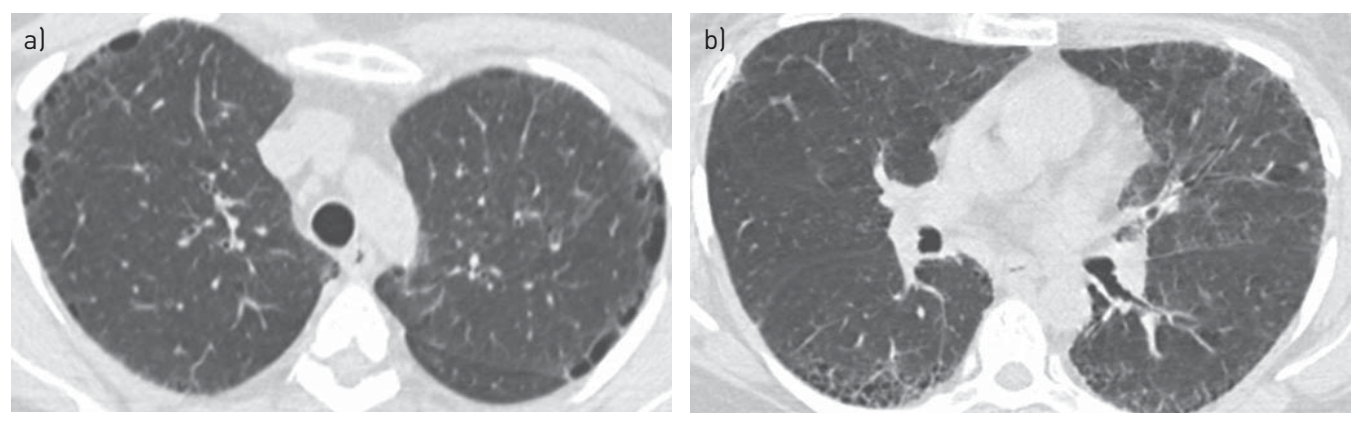

FIGURE 1 Chest high-resolution computed tomography scans. Multi-slice computed tomography demonstrates a) apical emphysema and b) honeycombing and reticulation with subpleural, basal predominance. Extensive ground-glass opacities can be seen in both panels. Image courtesy of Claus P. Heussel (Thoraxklinik, University of Heidelberg, Heidelberg, Germany). 
TABLE 1 Pulmonary function tests

\begin{tabular}{lcc} 
Parameter & Absolute & \% Predicted \\
\hline FVC L & 2.5 & 81 \\
FEV 1 L & 2.1 & 82 \\
FEV 1 FVC \% & 84 & 106 \\
RV L & 2.2 & 124 \\
TLC L & 4.7 & 94 \\
DLCO-SB & & 36 \\
DLCO-VA & & 54
\end{tabular}

FVC: forced vital capacity; FEV1: forced expiratory volume in $1 \mathrm{~s}$; RV: residual volume; TLC: total lung capacity; DLCO: diffusing capacity of the lung for carbon monoxide; SB: single breath; VA: alveolar volume.

\section{Commentary}

This case illustrates the complexity faced by clinicians in the management of ILD patients in the real-life setting and raises a number of points outside of the clinical guidelines [10]. These include challenges in the diagnosis of IPF, such as the possibility of earlier diagnosis through auscultation for characteristic "velcro" crackles by general practitioners and issues in diagnosing IPF on HRCT [11]. The use of a MDT in such situations may overcome some of the problems related to ILD diagnosis. This case serves as an example of this as extensive ground-glass opacities should, in theory, have ruled out IPF according to the current guidelines. However, in this patient the ground-glass opacities corresponded to another smoking-related ILD. Extensive interdisciplinary discussion was key in exploring the potential differential diagnoses. Furthermore, the MDT meetings helped formulate and implement an effective management approach; for example, initiation of smoking cessation to manage the smoking-related ILD, namely DIP.

Selecting which treatments to prioritise for IPF may be challenging in the real-life setting. Treatments to consider include preventative care (such as vaccinations), symptom-based medications, and nonpharmacological approaches (such as patient support groups and sports). In addition, the physician may be faced with decisions relating to the treatment of comorbidities and the optimal time to initiate drug treatment. As patients are becoming increasingly well informed through sources such as the internet, they often want clinicians to explain which treatment has been selected for their condition and why.

In this case study there was an unexpected decline in lung function, which may have been caused by a number of conditions such as an acute exacerbation of IPF, lack of patient compliance with treatment or untreated comorbidities. In actual fact, the lung function was compromised through a neurological disease leading to back pain. In the real-life setting, the differentiation of stable disease from progressive fibrosis can also be complicated due to the variable course of IPF and associated conditions.

In summary, the $2011 \mathrm{ATS} / \mathrm{ERS} / \mathrm{JRS} / \mathrm{ALAT}$ guidelines provide an evidence-based approach to the diagnosis and management of IPF but cannot cover the full spectrum of challenges associated with managing IPF patients in the clinical practice. First and foremost, the physician should aim for the earliest possible diagnosis of IPF. Decisions relating to preventative treatments, management of comorbidities and treatment side-effects, drug initiation/termination, and, ultimately, palliative care should be tailored to the patient.

\section{Case 2}

Managing an unusual case of IPF

IPF may be diagnosed in the context of various clinical backgrounds and the complex pathophysiology of this condition can sometimes present unusual or challenging situations in individual cases.

A 79-year-old male with a 45 pack-year smoking history first presented in February 2000 with chronic cough and rhinosinusitis. This was diagnosed clinically and histologically as cryptogenic organising pneumonia (COP). The patient was treated with a 6-month course of systemic steroids and the patient's cough completely subsided. In addition, previous consolidation on computed tomography disappeared; however, mild septal thickening persisted.

The patient was next seen in May 2006 for pre-operative assessment (transurethral resection) during which physical examination and chest radiographs were unremarkable. PFTs were relatively normal and similar to those observed in 2000; however, a decline in DLCO from 100\% in 2000 to $76 \%$ in 2006 was noted (table 2).

By the third consultation in September 2011, the patient had developed symptoms of typical ILD and had cancelled his gym membership 2 months earlier (July 2011) due to slowly emerging dyspnoea on exertion. 


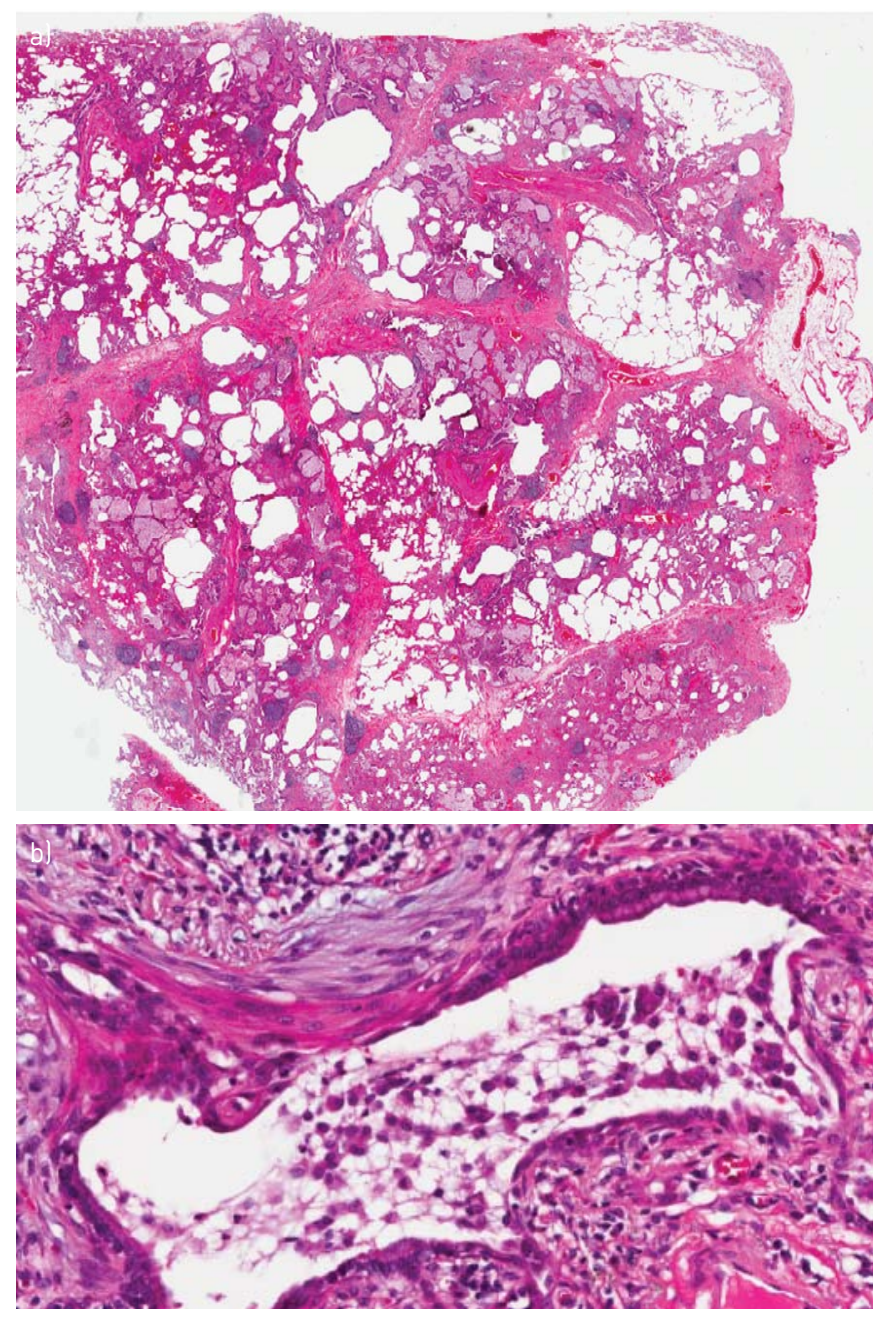

FIGURE 2 a) Low magnification histopathological biopsy showing typical features of usual interstitial pneumonia pattern with a heterogeneous appearance and areas of fibrosis with scarring and honeycomb change. Areas with less affected parenchyma also show emphysematous changes. b) Higher magnification of the histopathological biopsy reveals diffuse pulmonary involvement of numerous macrophage accumulations within most of the distal airspaces, consistent with a desquamative interstitial pneumonia pattern. Image courtesy of Philipp A. Schnabel (Institute of Pathology, University of Heidelberg, Heidelberg, Germany).

The patient had a productive cough (one tablespoon of sputum in $24 \mathrm{~h}$ ) and a 6-min walk test distance of $300 \mathrm{~m}$. Initial treatment included clarithromycin and ciprofloxacin but both were terminated early due to side-effects. Mild digit clubbing and dorsobasal velcro crackles were noted during physical examination. The patient was also found to have post-thrombotic syndrome (which had also been seen earlier in his history) along with lower limb pitting oedema. PFTs showed a further reduction in DLCO from 2006 and decreases in FVC, forced expiratory volume in $1 \mathrm{~s}$ (FEV1) and VC; FEV1/VC was 65\% (table 2).

Bronchoalveolar lavage (BAL) was performed but was technically biased due to the patient's cough. BAL findings included a predominance of macrophages (97\%) without significant lymphocytosis (3\%), ciliated epithelial cells and detritus. HRCT showed basal fibrosis and honeycombing. SLB was not performed since all the HRCT criteria for UIP pattern, as specified by the 2011 ATS/ERS/JRS/ALAT guidelines, were fulfilled [5]. The patient was diagnosed with IPF by the MDT and started treatment with full-dose pirfenidone $\left(2403 \mathrm{mg} \cdot \mathrm{day}^{-1}\right)$ and a PPI due to reflux symptoms.

In January 2011, the patient was admitted with an infrarenal aortic aneurysm which was stented without complication. At follow-up, the patient's cough had resolved and his dyspnoea on exertion had improved sufficiently for him to resume his gym membership. PFTs performed in October 2012 revealed stabilisation of DLCO (51.2\%) and higher VC, FVC and FEV1 values in comparison to October 2011 (table 2). An improvement was also seen on the chest HRCT scan in 2012 compared to the scan performed in 2011 


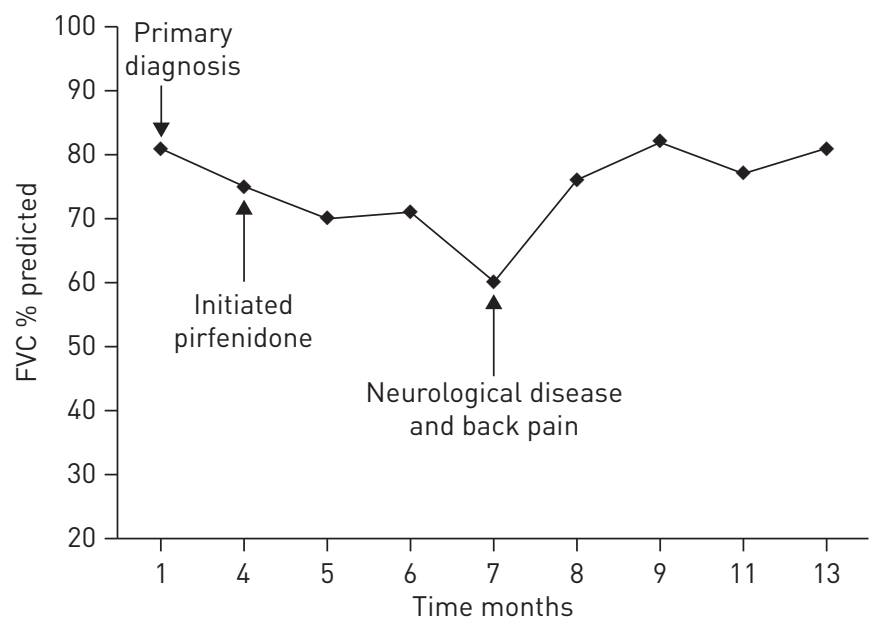

FIGURE 3 Forced vital capacity (FVC) over the course of the disease, some disease stabilisation can be seen following initiation of pirfenidone therapy. However, there was a significant decline in FVC due to neurological disease. After treatment for this disease the patient's FVC increased again.

(fig. 4). At the most recent consultation in 2013 no significant changes were observed in the PFTs compared to October 2012, except for a decreased DLCO. The patient was also noted to have more pronounced finger clubbing.

\section{Commentary}

The patient's age, smoking history, clinical presentation and radiology findings are consistent with the diagnosis of IPF [5], which was also concluded in a MDT discussion. However, this case has several unusual features. Both the initial presentation of COP in 2000 preceding IPF and its resolution upon cessation of corticosteroids was remarkable. Still, very limited reports on the progression of COP to fibrosis with honeycombing have been described [12] and it has to be kept in mind that in rare patients acute exacerbation of IPF may comprise organising pneumonia at lung biopsy [13]. However, the clinical course of this patient does not support the hypothesis of chronic COP as the underlying disease results in fibrotic lung disease or an initial acute exacerbation of an IPF with radiological features of COP. Moreover, the concurrent diagnosis of COP and IPF has been described previously [14]. In addition, a FVC/FEV1 of 67\%, although within the lower limit of normal, is uncharacteristically low for an IPF patient without emphysema, which was not present in this patient, and might account for some bronchiolar involvement [15]. The treatment of the infrarenal aortic aneurysm also presented a dilemma regarding whether it was better to treat the aneurysm with general anaesthesia and open surgery, which can further stress the patient, or to stent the aneurysm under local anaesthesia and risk an increased immune response.

Besides the unusual features of this case, the improvement in lung function with pirfenidone treatment was noticeable and has only rarely been reported to date. This is supported by the results of randomised controlled trials on pirfenidone reported to date [16-18]. In these trials some similar individual cases have

\section{TABLE 2 Pulmonary function tests performed between 2000 and $2012^{\#}$}

Date of test

Feb 1, 2000 May 29, $2006 \quad$ Oct 31, $2011 \quad$ April 21, $2012 \quad$ Sept 15, 2012 Oct 27, 2012

\begin{tabular}{lccccc}
\hline VC \% predicted & 100 & 101 & 80 & 88 & 88 \\
FVC \% predicted & 101 & 103 & 80 & 94 & 91 \\
FEV1\% predicted & 95 & 98 & 72 & 93 & 91 \\
FEV1/VC \% predicted & 71 & 71 & 65 & 76 & 92 \\
DLC0 \% predicted & 100 & 76 & 32 & 42 & 67 \\
\hline
\end{tabular}

VC: vital capacity; FVC: forced vital capacity; FEV1: forced expiratory volume in $1 \mathrm{~s}$; DLCo: diffusing capacity of the lung for carbon monoxide. ${ }^{\#}$ : the full dose of pirfenidone was started in November 2011. 

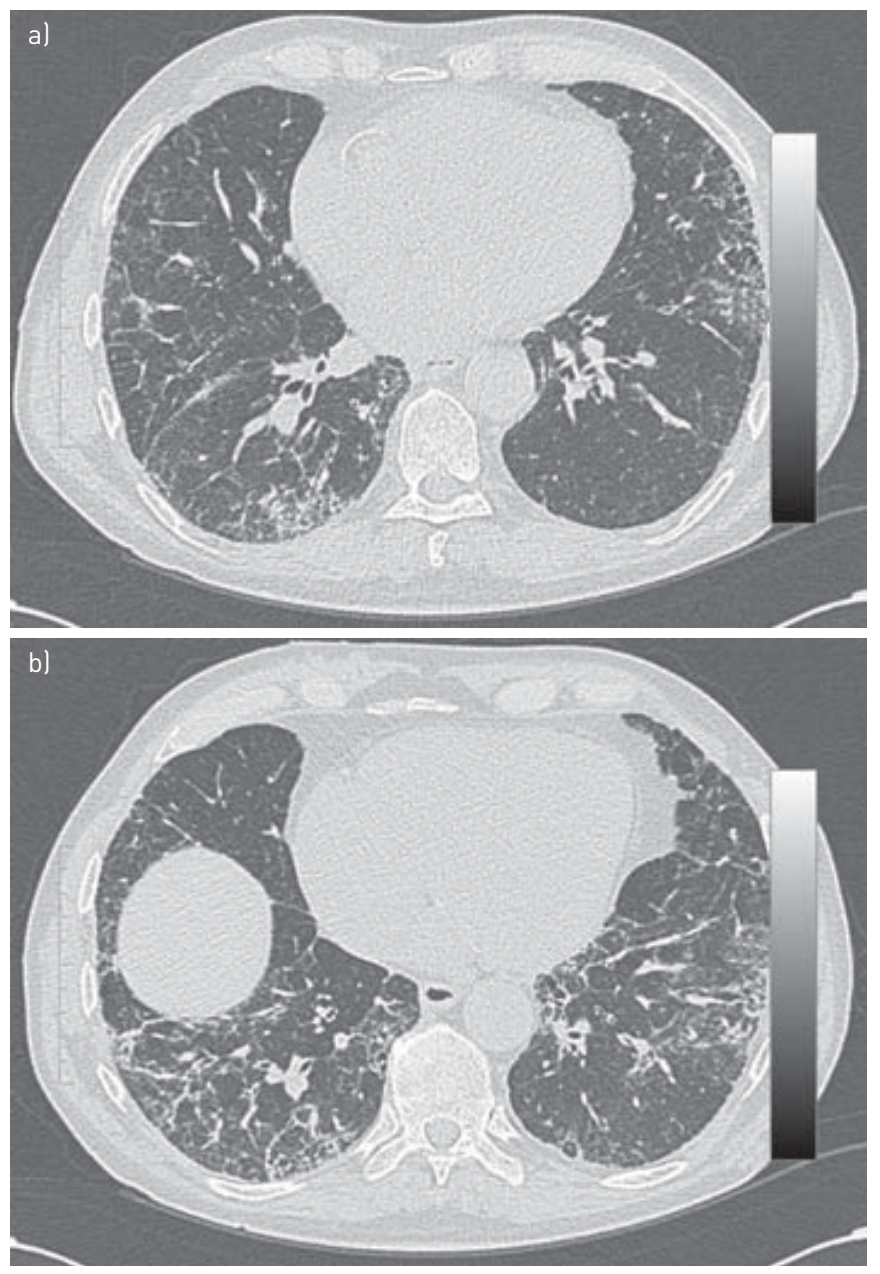

FIGURE 4 Chest high-resolution computed tomography scans performed on a) August 16, 2011 and b) September 4, 2012.

been described. However, the underlying pathophysiology of this interesting observation is not fully understood and should be explored further.

\section{Case 3}

Atypical accelerated UIP: a special challenge?

Some patients with IPF follow a relatively rapid decline but it is not known if this represents a distinct phenotype. Further characterisation of these patients could be important, particularly regarding earlier diagnosis and early treatment.

A 73-year-old retired, male executive had a cough which persisted for 3 months and was unresponsive to antibiotics. The patient was an ex-smoker with a 40 pack-year smoking history and his past medical history included coronary artery disease for which he underwent stenting procedures in 2005 and 2007. The patient did not, however, receive amiodarone for this condition. Investigations included a chest radiograph which was followed by a HRCT scan, both of which showed irregular results.

The patient was first referred to the respiratory clinic in 2010. On examination, the patient appeared relatively well with no shortness of breath or finger clubbing; however, minimal bi-basal, fine, endrespiratory crackles were present during auscultation. Serology testing (extractable nuclear antigens screen and anti-cyclic citrullinated peptides) was negative but initial PFTs revealed a number of low parameters including total lung capacity (72\% predicted), VC (72\% pred), FVC (69\% pred) and DLCO (61\% pred). Resting oxygen saturation was normal (96\%) but low under exertion (91\%) (table 3). HRCT showed mild reticulation and sub-pleural honeycombing (fig. 5).

The patient was diagnosed with IPF which was followed by a progressive decline in lung function with worsening PFTs and HRCT scans. 
TABLE 3 Initial pulmonary function tests performed in November 2011

\begin{tabular}{|c|c|c|c|c|}
\hline & \multicolumn{2}{|c|}{ Predicted } & \multicolumn{2}{|c|}{ Patient } \\
\hline & Normal & Range & Measured & $\%$ Predicted \\
\hline \multicolumn{5}{|l|}{ Lung volumes } \\
\hline TLCpleth L & 6.44 & $>5.07$ & $4.61^{\#}$ & 72 \\
\hline VC L & 4.13 & $>3.30$ & $2.97^{\#}$ & 72 \\
\hline RV L & 2.30 & $<2.99$ & 1.64 & 71 \\
\hline $\mathrm{RV} / \mathrm{TLC}$ & 35.8 & $<46.9$ & 35.6 & 100 \\
\hline FRC L & & & 2.2 & \\
\hline \multicolumn{5}{|l|}{ Spirometry } \\
\hline FVC L & 4.13 & $>3.30$ & $2.86^{\#}$ & 69 \\
\hline FEV1 L & 3.19 & $>2.51$ & $2.30^{\#}$ & 72 \\
\hline FEV1/FVC \% & 77.1 & $>67.9$ & 80.4 & \\
\hline FEF $25-75 \mathrm{~L} \cdot \mathrm{S}^{-1}$ & 2.8 & $>1.5$ & 2.1 & 75 \\
\hline $\mathrm{FEFmax} L \cdot \mathrm{S}^{-1}$ & 7.8 & $>4.4$ & 11.0 & 142 \\
\hline $\mathrm{FIFmax} \mathrm{L} \cdot \mathrm{S}^{-1}$ & & & 3.7 & \\
\hline FEF50/FIF50 & & & 0.7 & \\
\hline$M V V L \cdot \min ^{-1}$ & 124 & $>91$ & $87^{\#}$ & 71 \\
\hline \multicolumn{5}{|l|}{ Diffusing capacity } \\
\hline $\mathrm{DLCO}-\mathrm{SB} \mathrm{L} \cdot \mathrm{min}^{-1} \cdot \mathrm{mmHg}^{-1}$ & 25.3 & $>17.3$ & $15.4^{\#}$ & 61 \\
\hline DLco adjusted for $\mathrm{Hb}$ & \multicolumn{2}{|c|}{$16.0 \mathrm{~g} \cdot \mathrm{dL}^{-1}$} & $14.8^{\#}$ & 59 \\
\hline VA L & 6.23 & $>5.07$ & $3.82^{\#}$ & 61 \\
\hline \multicolumn{5}{|l|}{ Oximetry } \\
\hline Oxygen saturation $\%$ & 96 & $\geqslant 93$ & 96 & \\
\hline \multicolumn{5}{|l|}{ Heart rate } \\
\hline Pulse beats $\cdot \mathrm{min}^{-1}$ & & & 70 & \\
\hline
\end{tabular}

TLCpleth: total lung capacity (TLC) measured by plethysmography; VC: vital capacity; RV: residual volume; FRC: functional residual capacity; FVC: functional vital capacity; FEV1: forced expiratory volume in $1 \mathrm{~s}$; FEF25-75: forced expiratory flow at 25-75\% of FVC; FEFmax: maximal forced expiratory flow; FIFmax: maximum forced inspiratory flow; FEF50: forced expiratory flow at 50\% of FVC; FIF50: forced inspiratory flow at 50\% of FVC; MVV: maximum voluntary ventilation; DLCO: diffusing capacity of the lung for carbon monoxide; SB: single breath; $\mathrm{Hb}$ : haemoglobin; VA: alveolar volume. ${ }^{\#}$ : outside normal limits.

In December 2012, the patient started pirfenidone therapy and disease progression was reduced or stabilised according to PFTs (fig. 6) and HRCT scans (fig. 7) for a period of 6 months. However, he subsequently deteriorated and was placed on the lung transplantation list. Following this, in July 2013, he suffered an acute exacerbation of IPF for which he was admitted and treated but died shortly afterwards.

Commentary

IPF is a disease with a highly variable course. Currently, there is no reliable marker to predict which patients may exhibit a more rapidly progressive course. Therefore, the only way to identify such patients is to follow their pulmonary function parameters (FVC, DLCO and 6-min walking distance) and, potentially, in some patients radiological examinations (i.e. HRCT) over time. However, by the time the physician recognises that the decline represents an accelerated (and irreversible) progression of the disease, rather than biological variability or acute exacerbations, it may already be too late to intervene in the progressive fibrotic process. Still, one might argue that early identification of rapid progressors is mainly important for lung transplant listing and with regards to prognosis but not for pharmacological treatment because there were no adverse treatment implications of not "catching the disease early".

Since the approval of pirfenidone and perhaps with other potential treatments on the horizon, this now raises the question as to whether it is critically important from a therapeutic perspective to make a more rapid diagnosis and start pharmacological treatment earlier. This is an attractive possibility, but at this point it is only a hypothesis with no supporting evidence. Analysing the available evidence from the CAPACITY trials does not suggest that pirfenidone is more effective in patients with mild, early disease or that it is effective in more severe disease [16]. The beneficial effect of pirfenidone, as demonstrated in randomised, double-blind, placebo-controlled trials, was not dependent on patients being randomised during a progressive period. Personal experience and currently unpublished data raise the possibility that pirfenidone may contribute to disease stabilisation in slightly more progressive phenotypes. In the absence of a defined 

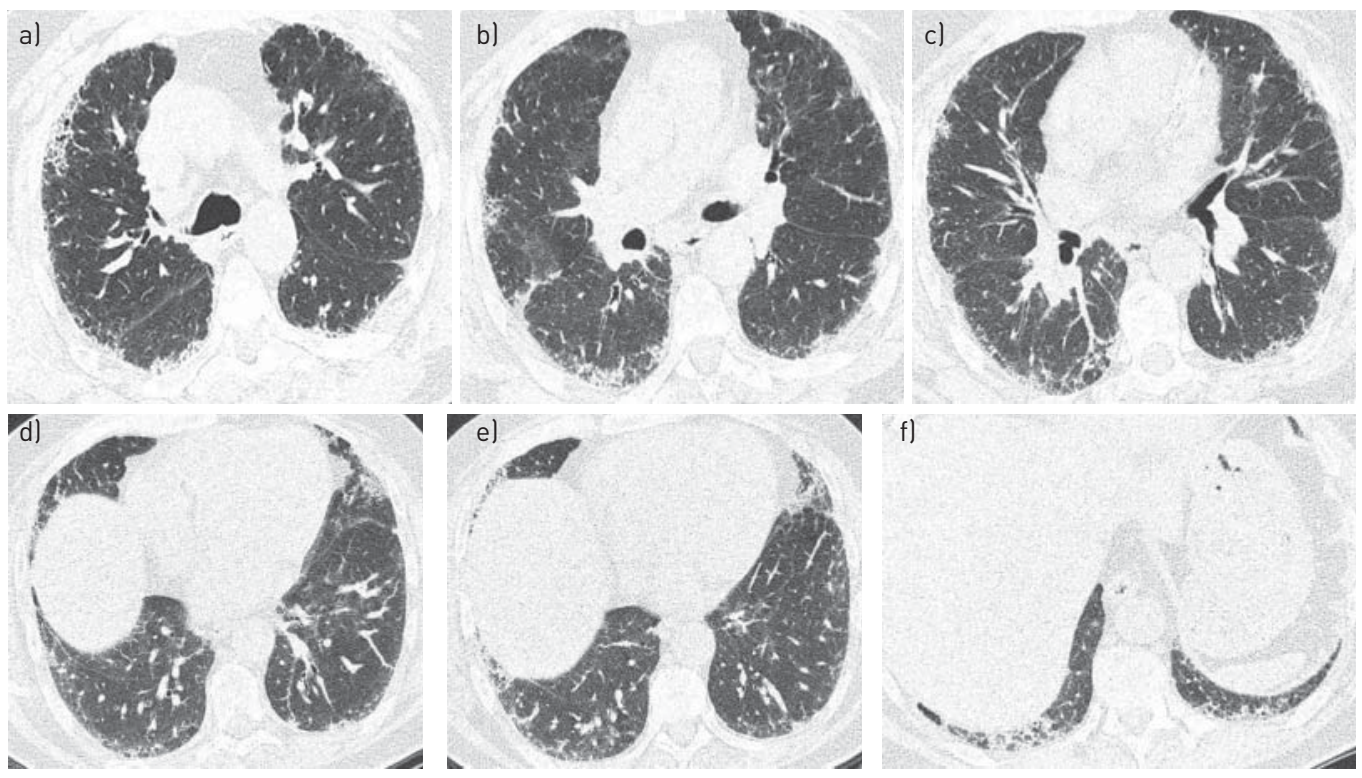

FIGURE 5 Initial high-resolution computed tomography scans performed in November 2011 showing mild reticulation and sub-pleural honeycombing. Representative sections of the lungs from a) the upper to f) the lower zones.

"pirfenidone-responsive phenotype", individual decisions on the optimal time to start treatment need to be made. While comparison of pre- and post-treatment trajectories of FVC decline may represent an attractive option to monitor disease progression, the highly variable and unpredictable nature of disease progression in IPF makes it difficult to assess treatment response in individual patients.

This was our intention in initiating treatment with pirfenidone in this case. It is interesting that examination of pulmonary function data, as well as clinical follow-up, suggests that initially there was a beneficial response to pirfenidone treatment. We noted a tendency for improvement in FVC, 6-min walking distance and oxygen saturations. However, despite receiving treatment with pirfenidone, the patient
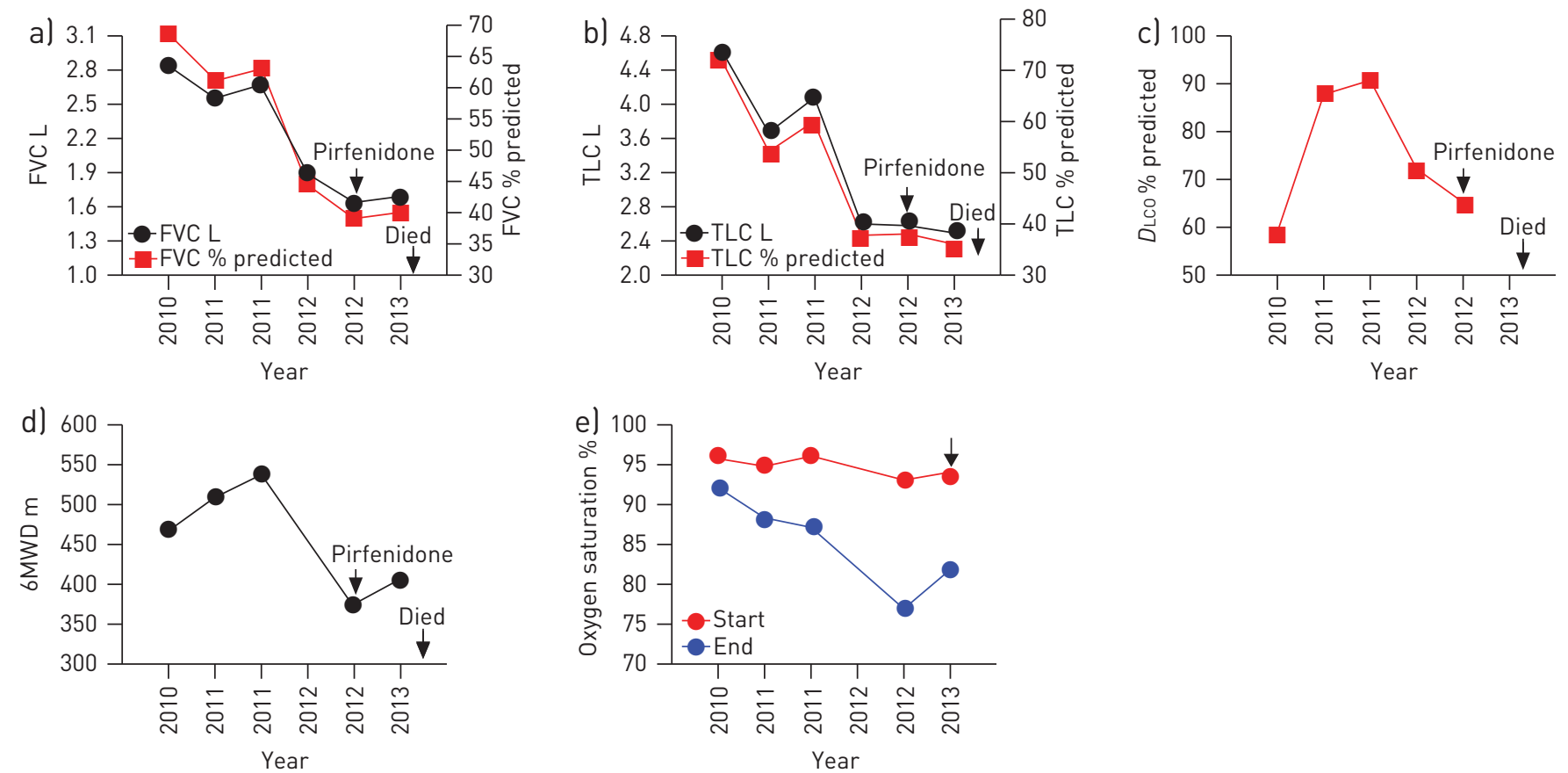

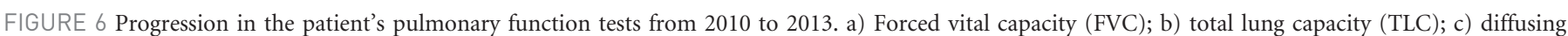

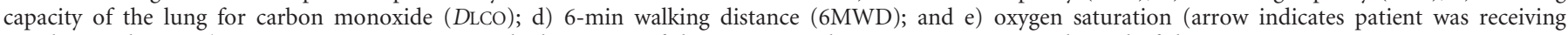
supplemental oxygen). Start: oxygen saturation at the beginning of the 6MWD; end: oxygen saturation at the end of the 6MWD. 

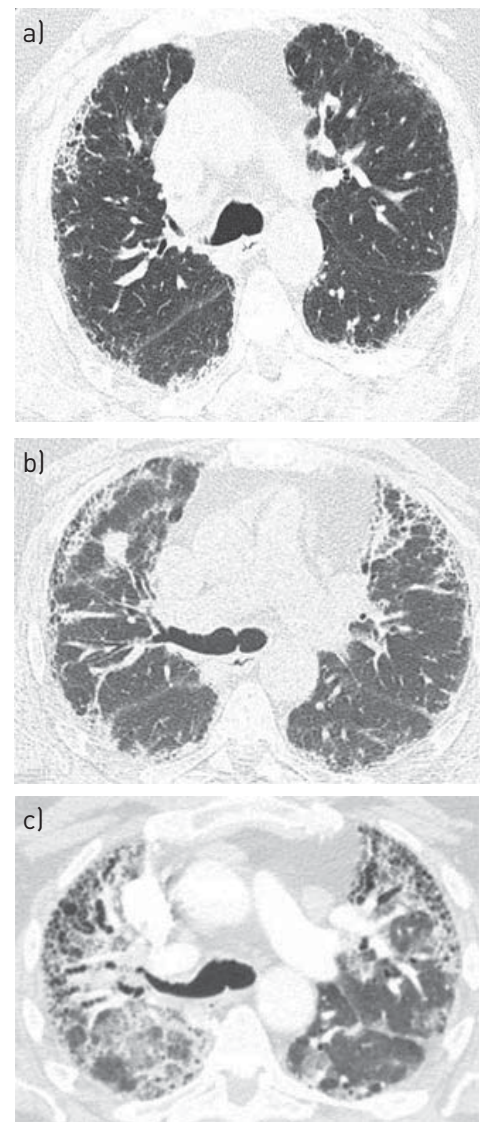
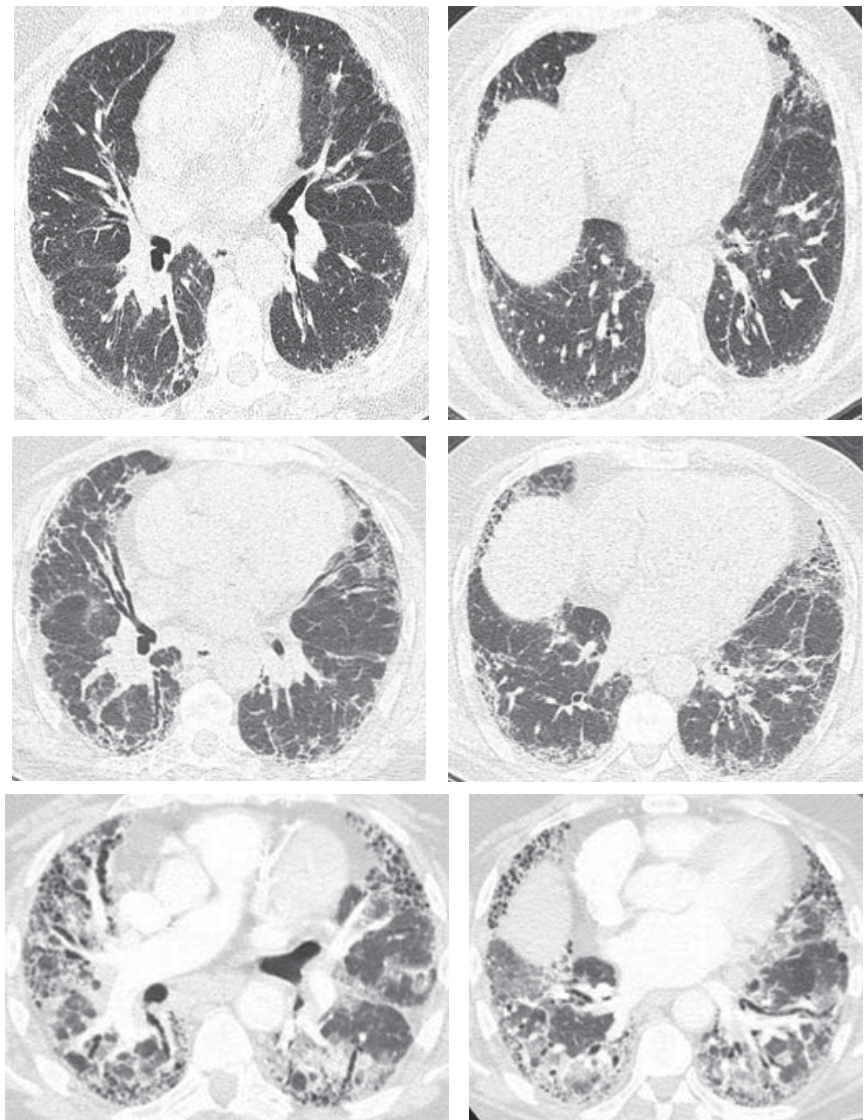

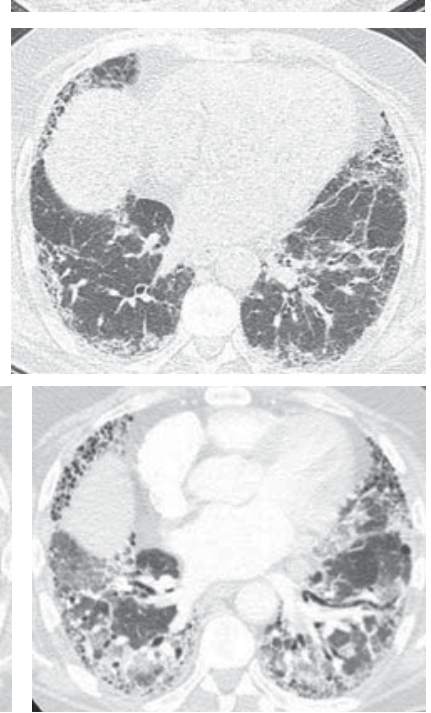

FIGURE 7 Patient computed tomography scans performed in a) 2010, b) 2012 and c) 2013. Representative sections of the lungs from the upper region to the lower region (left to right).

developed a fatal exacerbation of IPF. Whether earlier institution of pirfenidone would have stabilised (or improved) his pulmonary function is not yet supported by data and clinical trials with pirfenidone do not demonstrate prevention of acute exacerbations [16]. We await publication of further clinical data with pirfenidone, such as the ASCEND trial, which may further help to answer some of these questions, such as when it may be best to start treatment with pirfenidone, in which types of patients, the magnitude of benefit in terms of stabilising lung function, and evidence for future treatment modalities that may reduce the risk of exacerbations.

\section{Acknowledgements}

The author contributions are as follows. Case 1: Michael Kreuter; Case 2: Peter Kardos; and Case 3: Victor Hoffstein.

This article is based on the proceedings of the 2013 Advancing IPF Research (AIR) meeting (Nice, France), which was sponsored by InterMune International AG (Muttenz, Switzerland). Medical writing support was provided by Michael Smith (IntraMed International, Milan, Italy), which was funded by InterMune International AG.

\section{References}

Meltzer EB, Noble PW. Idiopathic pulmonary fibrosis. Orphanet J Rare Dis 2008; 3: 8.

2 Kim DS, Collard HR, King TE Jr. Classification and natural history of the idiopathic interstitial pneumonias. Proc Am Thorac Soc 2006; 3: 285-292.

3 Collard HR, Moore BB, Flaherty KR, et al. Acute exacerbations of idiopathic pulmonary fibrosis. Am J Respir Crit Care Med 2007; 176: 636-643.

4 Nagai S, Nagao T, Kitaichi M, et al. Clinical courses of asymptomatic cases with idiopathic pulmonary fibrosis and a histology of usual interstitial pneumonia. Eur Respir J 1998; 12: Suppl. 28, 131 s.

5 Raghu G, Collard HR, Egan JJ, et al. An official ATS/ERS/JRS/ALAT statement: idiopathic pulmonary fibrosis: evidence-based guidelines for diagnosis and management. Am J Respir Crit Care Med 2011; 183: 788-824.

6 Cottin V. The role of pirfenidone in the treatment of idiopathic pulmonary fibrosis. Respir Res 2013; 14: Suppl. 1, S5.

7 Kreuter M. Pirfenidone: an update on clinical trial data and insights from everyday practice. Eur Respir Rev 2014; 23: 111-117.

8 Doyle TJ, Hunninghake GM, Rosas IO. Subclinical interstitial lung disease: why you should care. Am J Respir Crit Care Med 2012; 185: 1147-1153. 
Cottin V, Nunes H, Brillet PY, et al. Combined pulmonary fibrosis and emphysema: a distinct underrecognised entity. Eur Respir J 2005; 26: 586-593.

10 Oltmanns U, Kahn N, Palmowski K, et al. Pirfenidone in idiopathic pulmonary fibrosis: real life experience from a German tertiary referral center for interstitial lung diseases. Respiration 2014 (in press).

11 Cottin V, Cordier JF. Velcro crackles: the key for early diagnosis of idiopathic pulmonary fibrosis? Eur Respir J 2012; 40: 519-521.

12 Cottin V, Cordier JF. Cryptogenic organizing pneumonia. Semin Respir Crit Care Med 2012; 33: 462-475.

13 Dallari R, Foglia M, Paci M, et al. Acute exacerbation of idiopathic pulmonary fibrosis. Eur Respir J 2004; $23: 792$.

14 Nagata N, Nagatomo H, Yoshii C, et al. Features of idiopathic pulmonary fibrosis with organizing pneumonia. Respiration 1997; 64: 331-335.

15 Egan J, Martinez F, Wells A, et al. Lung function estimates in idiopathic pulmonary fibrosis: the potential for a simple classification. Thorax 2005; 60: 270-273.

16 Noble PW, Albera C, Bradford WZ, et al. Pirfenidone in patients with idiopathic pulmonary fibrosis (CAPACITY): two randomised trials. Lancet 2011; 377: 1760-1769.

17 Taniguchi H, Ebina M, Kondoh Y, et al. Pirfenidone in idiopathic pulmonary fibrosis. Eur Respir J 2010; 35: 821-829.

18 Azuma A, Nukiwa T, Tsuboi E, et al. Double-blind, placebo-controlled trial of pirfenidone in patients with idiopathic pulmonary fibrosis. Am J Respir Crit Care Med 2005; 171: 1040-1047. 